\title{
Indium Phosphide-Based Semiconductor Nanocrystals and Their Applications
}

\author{
Paul Mushonga, ${ }^{1}$ Martin O. Onani, ${ }^{1}$ Abram M. Madiehe, ${ }^{2}$ and Mervin Meyer ${ }^{2}$ \\ ${ }^{1}$ Department of Chemistry, University of the Western Cape, Private Bag X17, Bellville 7535, South Africa \\ ${ }^{2}$ Department of Biotechnology, University of the Western Cape, Private Bag X17, Bellville 7535, South Africa
}

Correspondence should be addressed to Martin O. Onani, monani@uwc.ac.za

Received 3 July 2011; Revised 25 October 2011; Accepted 26 October 2011

Academic Editor: Marinella Striccoli

Copyright (C) 2012 Paul Mushonga et al. This is an open access article distributed under the Creative Commons Attribution License, which permits unrestricted use, distribution, and reproduction in any medium, provided the original work is properly cited.

\begin{abstract}
Semiconductor nanocrystals or quantum dots (QDs) are nanometer-sized fluorescent materials with optical properties that can be fine-tuned by varying the core size or growing a shell around the core. They have recently found wide use in the biological field which has further enhanced their importance. This review focuses on the synthesis of indium phosphide (InP) colloidal semiconductor nanocrystals. The two synthetic techniques, namely, the hot-injection and heating-up methods are discussed. Different types of the InP-based QDs involving their use as core, core/shell, alloyed, and doped systems are reviewed. The use of inorganic shells for surface passivation is also highlighted. The paper is concluded by some highlights of the applications of these systems in biological studies.
\end{abstract}

\section{Introduction}

Spherical semiconductor nanocrystals, which may be referred to simply as quantum dots (QDs), are nanometersized clusters that are generally composed of a few hundreds to several thousands of atoms from Groups II-VI, III-V, and IV-IV [1-5]. The physical dimensions of QDs are on the scale of the excitonic Bohr radius, a property that leads to a phenomenon called the quantum confinement effect (QCE), whose origins are explained by Andersen et al. using II-VI nanocrystallites [6]. The QCE leads to tunable optical and electronic properties that are not observed in either the bulk solids or the molecular level.

The QDs have unique and distinctive optical properties in comparison to organic dyes and fluorescent protein molecules. These properties [7] include (i) broad absorption spectra that increase towards the ultra violet (UV) region from the first absorption band edge; (ii) very large molar extinction coefficients in the order of $0.5-5 \times 10^{6} \mathrm{M}^{-1} \mathrm{~cm}^{-1}$, about 10-50 times larger than those of organic fluorophores, making them brighter probes suitable for studies involving in vivo investigations, where light intensities are severely attenuated by absorption and scattering; (iii) longer excited state lifetimes and hence large effective Stokes shifts enabling the separation of QD fluorescence from the background fluorescence; (iv) large surface area-to-volume ratio which allows them to be conjugated to various molecules; (v) high photostability, a versatile property that facilitates visualization of biological material for a longer period; and (vi) narrow and symmetrical emission spectra that allow various colors to be distinguished without any spectral overlap.

\section{Quantum Dots of Group III-V}

For more than two decades, research into semiconductor nanocrystals has been focused on the fabrication of versatile groups II-VI QDs owing to their potential application in lasers [8], light-emitting diodes [9], and more importantly biological studies [10-12]. However, due to the presence of highly toxic cadmium in the design, their biological application has been limited. In order to alleviate this toxicity problem, a process of overcoating the cadmium-based core with a less toxic shell such as $\mathrm{ZnS}$ was developed. In this regard, the shell would prevent leakage of the toxic ions. Unfortunately this did not make the II-VI QDs completely 
TABle 1: Band gap $\left(E_{g}\right)$ and excitonic Bohr radius $\left(r_{B}\right)$ in some semiconductors.

\begin{tabular}{lccc}
\hline Compound & Band gap $(\mathrm{eV})$ & $r_{B}(\mathrm{~nm})$ & Reference \\
\hline InP & 1.35 & 15 & {$[17]$} \\
InAs & 0.354 & 34 & {$[18]$} \\
InSb & 0.17 & 65.6 & {$[19]$} \\
CdS & 2.43 & 5.8 & {$[20]$} \\
CdSe & 2.87 & 5.3 & {$[21]$} \\
CdTe & 1.5 & 7.3 & {$[22]$} \\
ZnSe & 2.67 & 4.5 & {$[23]$} \\
\hline
\end{tabular}

innocuous as exposure to UV light or oxidation as a result of inflammatory responses induces the release of cadmium via surface oxidation [13]. The focus in the last two decades was therefore shifted towards the fabrication of III-V QDs in general, and InP in particular. The principal attraction to these semiconductors lies in the robustness of the covalent bond found in groups III-V semiconductor matrix compared to the ionic bond in the groups II-VI semiconductors. The presence of the covalent bond serves two functions, which are the enhancement of the optical stability of the QD systems as well as the reduction of the toxicity as a result of the nonerosion of the constituent species when used in biological milieu $[14,15]$. The other reason for the interest in the III-V systems lies in the fact that the excitonic Bohr radius is larger in III-V than in the II-VI systems (Table 1). Consequently, groups III-V QDs exhibit stronger size quantization effects in comparison with the groups II-VI systems [16].

In order for these QD to display their superior properties well, they ought to be monodispersed. The surfactant organic molecules are usually employed for this purpose because they bind to the QD core during the synthesis effectively preventing their aggregation [24]. The surfactant layer also has the ability to control the size and shape of the growing QDs. The layer provides imperfect passivation of the nanocrystal surface dangling bonds due to the lability of the organic ligands [25]. The resultant defects, also referred to as surface traps, act as sites for nonradiative decay of the QD excited state [26]. Some of the excited state electrons can first decay to the surface states lying in the mid band gap and then recombine nonradiatively with the holes in the valence band, reducing the photoluminescent quantum yield. Several techniques have been employed to enhance the photoluminescence quantum yield. The effective strategies involve epitaxially growing an inorganic shell of a large band gap material around each particle, or just chemically modifying the particle surface. Sometimes overcoating the nanocrystal core with an inorganic shell of a higher band gap semiconductor effectively passivates surface-related defects resulting in an improved fluorescence quantum yield as well as enhanced photostability [27]. Kim et al. [28] reported that the emission efficiency of the InP/ZnSe core/shell was 6.8 times higher than that of the bare InP QDs, demonstrating that surface traps are effectively depressed after epitaxially depositing the larger band gap semiconductor ZnSe onto the InP nanocrystal core. The inorganic shell type and shell thickness are critical in the tailoring of the optical and electronic properties of the QDs [25]. Chemically modifying the surface of the InP QD core entails etching them with dilute ethanolic or butanolic solutions of $\mathrm{HF}$ [29] or $\mathrm{NH}_{4} \mathrm{~F}$ [30]. This etching process effectively removes phosphorus dangling bonds [31] culminating in an increase in near band edge emission by a large factor of 10 [29].

\section{General Synthetic Techniques}

The general synthesis of high-quality QDs is of utmost importance as all their properties are dependent on particle quality which is controlled by size distribution and crystal defects. There are generally two techniques, namely, the hotinjection and heating-up techniques which have evolved in the last two decades. These techniques have been established for the synthesis of the majority of the reported QDs [32].

3.1. Hot-Injection Technique. This technique involves the rapid injection of a room temperature solution of precursors into an extremely hot reaction medium in the presence of carefully chosen surfactant molecules. The rapid injection of the precursor solution induces the sudden supersaturation of the solution resulting in a short burst of nucleation. The injected solution reduces the reaction temperature and dilutes the concentration of reactants. With the reaction temperature dropping in response to addition of the cold precursor solution, coupled with the low concentration of the remaining precursor molecules, further nucleation is prevented. The eventual growth of the nanocrystals follows at a lower temperature than that of the nucleation process. This sequential separation of the nucleation and growth processes generally leads to a precise control of the size and shape of the semiconductor nanocrystals. This latter process forms the strength of this technique. A pioneering example of this technique was reported by Murray et al. [33]. They injected cadmium and selenium precursors into a hot $\left(300^{\circ} \mathrm{C}\right)$ solution of tri-n-octylphosphine oxide (TOPO). The growth temperature used was $230-260^{\circ} \mathrm{C}$. Here the surfactant acts in fourfold. Firstly, it is as a coordinating solvent that controls the growth process while at the same time stabilizing the nanocrystal core. Secondly, the surfactant binds to the surface of the nanocrystals providing a barrier to the addition of more material to the surface of the nanocrystals slowing down the growth kinetics [24]. Thirdly, it serves to prevent the aggregation of particles and finally passivates the surface of the nanocrystals.

3.2. Heating-up Technique. This is a batch process where all the precursors are mixed at room temperature followed by a rapid heating of the system to the appropriate growth temperature for the nanocrystals. There is no operation under this method that induces high supersaturation as in the hot-injection method. Under this technique, the supersaturation level and the temperature of the solution increase together, and the nucleation rate is sensitive to both [34]. The procedure allows for scaling up as well as improving reproducibility [35]. 


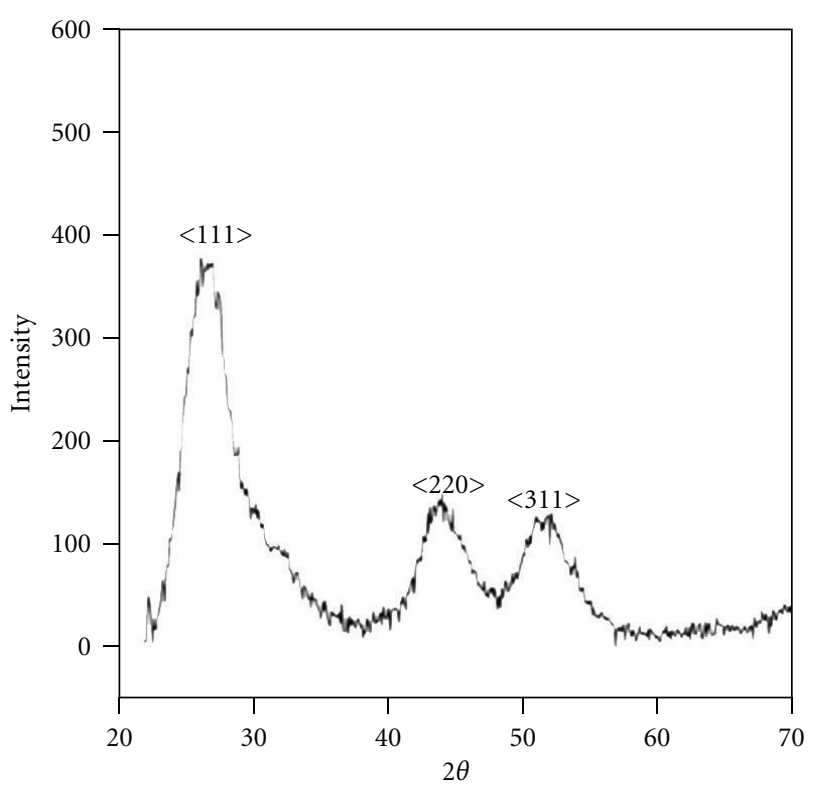

(a)

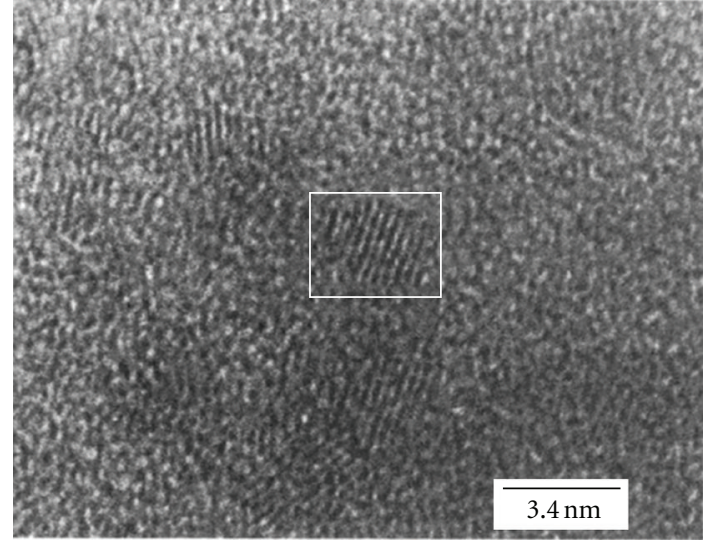

(b)

FIGURE 1: An X-ray diffraction pattern for dried InP QD exhibiting broadened prominent peaks for InP (a) and an HRTEM micrograph showing lattice fringes (b) (reprinted with permission from [36], Copyright 1994, American Chemical Society).

\section{Synthesis and Optical Properties of InP-Based QDs}

4.1. Building Core and Core/Shell InP QDs. The central part of a nanocrystal constitutes the core, and the immediate surrounding comprises the shell. In 1994, well-crystallized InP QDs with a zinc blende structure were for the first time synthesized [36]. A chloroindium oxalate complex (prepared from the reaction of indium trichloride and sodium oxalate) and tris(trimethylsilyl)phosphine $\left[(\mathrm{TMS})_{3} \mathrm{P}\right]$ were used as sources of indium and phosphorus, respectively. A mixture of TOPO and trioctylphosphine (TOP) was used as a stabilizer and the reaction was carried out at $270^{\circ} \mathrm{C}$ over three days. The nanocrystals were characterized by X-ray diffraction (XRD) and high-resolution transmission electron microscopy (HRTEM) (Figure 1). X-ray diffraction data on the InP nanocrystal powders showed the $\langle 111\rangle,\langle 220\rangle$, and $\langle 311\rangle$ peaks of crystalline zinc blende InP at $2 \theta$ values of $26.2 \pm 0.2^{\circ}, 46.3 \pm 0.2^{\circ}$, and $51.7 \pm 0.2^{\circ}$, respectively. HRTEM images showed that the particles were ellipsoidal rather than spherical in shape.

An optimization exercise for the properties of the obtained nanocrystals was done by changing the ratios of the In: P. A marked narrowing of the QD size distribution was obtained when indium was added in excess whereas the photoluminescence (PL) spectrum had two bands-one close to the band edge and the other above $800 \mathrm{~nm}$ (Figure 2) [37]. In the case of excess addition of phosphorus, only band edge emission was observed and the absorption spectrum did not show any resolved excitonic structure indicating a broad size distribution.

Further optimization was achieved by the etching process [31]. The etching of the QDs by using HF produced a photoluminescence emission spectrum consisting of a single narrow peak near the absorption band edge with quantum yield (QY) of about 30\% after the treatment. The InP QD cores with a lattice-matched $\mathrm{ZnCdSe}_{2}$ shell and photoluminescence QYs of $5-10 \%$ have also been synthesized [38]. However, it is worth noting that preetching the InP cores before shell growth results in poor core-shell structures as the HF blocks the surface of the QDs.

Kim et al. synthesized InP/CdSe core/shell type structure showing an increase in QY at the beginning of the overcoating process due to the small difference between the InP and CdSe conduction bands of $0.19 \mathrm{eV}$ which gives a type-I configuration but changes to type-II which is associated with loss of QY yield with increased shell thickening [39]. In such a synthesis, increase in the thickness of the shell should be avoided because it may result in a red shift, broadening of the full width at half maximum (FWHM), and a decrease in QYs. In 2009, InP/ZnS QDs with a diameter of 15-20 nm were reported [15]. Here, myristic acid was used as a stabilizer and octadecene as the noncoordinating solvent. PL studies showed that the QDs exhibited a band edge emission at around $650 \mathrm{~nm}$ with QY of 25-30\%. These InP/ZnS QDs were further functionalized using mercaptosuccinic acid rendering them highly dispersible in aqueous media. The mercaptosuccinic acid-capped QDs were stable for more than one week while dispersed in a common physiological buffer such as the phosphate buffer saline (PBS). Highly luminescent InP/ZnS nanocrystals were also synthesized following a single-step heating-up procedure [35]. Here, indium myristate, (TMS) ${ }_{3} \mathrm{P}$, zinc stearate, and dodecanethiol were used for the fabrication of the nanocrystals which gave high fluorescence quantum yields (50-70\%). These high PL QYs are attributed to the nanocrystals possessing a radial 


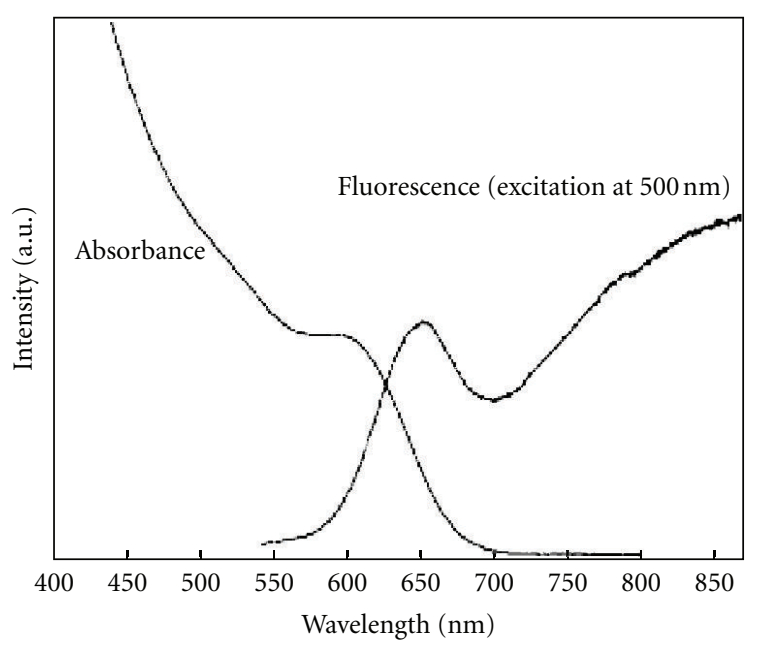

(a)

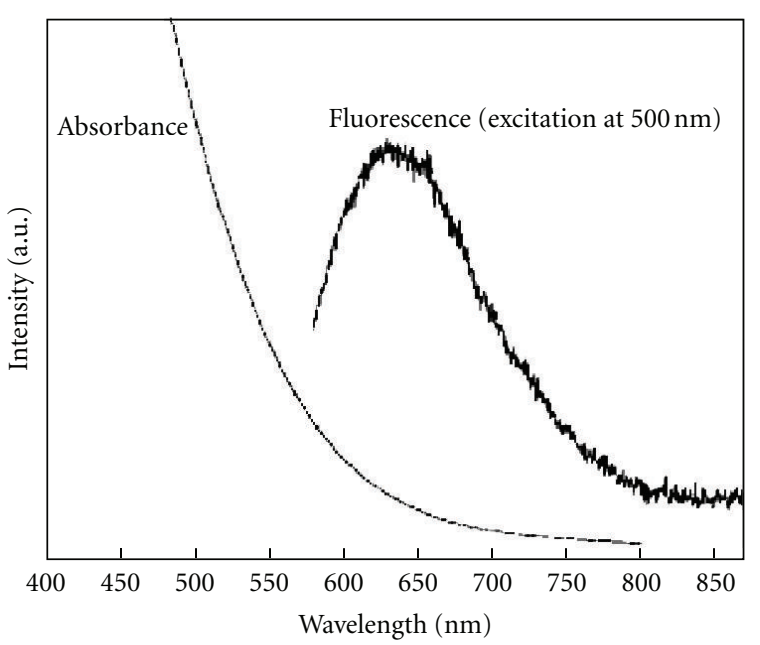

(b)

Figure 2: Room temperature absorption and PL spectra of InP QDs synthesized with different ratios of In and P: (a) In : P = 1.6; (b) In: P = 0.62 (reprinted with permission from [37], Copyright 2009, Elsevier).

composition gradient that relieves the strain due to the lattice mismatch between InP and $\mathrm{ZnS}$. A similar preparation was reported by another group but using stepwise process [40]. Again, palmitic acid was used as a stabilizer and octadecene as the noncoordinating solvent. The reaction between zinc acetate and palmitic acid gave that acetic acid causes the etching of the surface of the $\mathrm{InP}$ core leading to the easy formation of the shell. A blue shift in the emission and an improvement in quantum yield (38\%) were realized. The use of the poisonous $\mathrm{PH}_{3}$ gas, generated from the reaction of a metal phosphide (calcium phosphide) with $\mathrm{HCl}$ as a phosphorus source in InP QD synthesis, was also reported by Li et al. [41]. Again, octadecene and myristic acid were used as noncoordinating solvent and ligand, respectively. Unlike the single injection of (TMS) ${ }_{3} \mathrm{P}$, here the $\mathrm{PH}_{3}$ gas is continuously and slowly generated and added to the reaction system ensuring that the growth of the InP nanocrystals takes place in the size-focusing regime. The use of higher overall precursor concentrations resulted in the formation of a large number of nuclei and hence a smaller average size of the QDs. The PL spectra of the as-prepared InP nanocrystals showed two additional bands around 730 and $820 \mathrm{~nm}$. These bands were attributed to trap-state-related emissions, which are suppressed upon overcoating with $\mathrm{ZnS}$ obtained from the monomolecular zinc ethylxanthate precursor. The PL QY of up to $22 \%$ was achieved [41]. In a different experiment, the as-synthesized bare InP nanocrystals, prepared using $\mathrm{PH}_{3}$ gas, did not exhibit detectable photoluminescence owing to the presence of surface states [42]. However, when overcoated with a $\mathrm{ZnS}$ shell, a very strong PL intensity was observed, which was attributed to the passivation of these surface states by the inorganic shell. The emission profiles of the InP core varied as a function of size in the range of $600-700 \mathrm{~nm}$ while the $470 \mathrm{~nm}$ band is attributed to defect-related emissions from the shell (Figure 3).

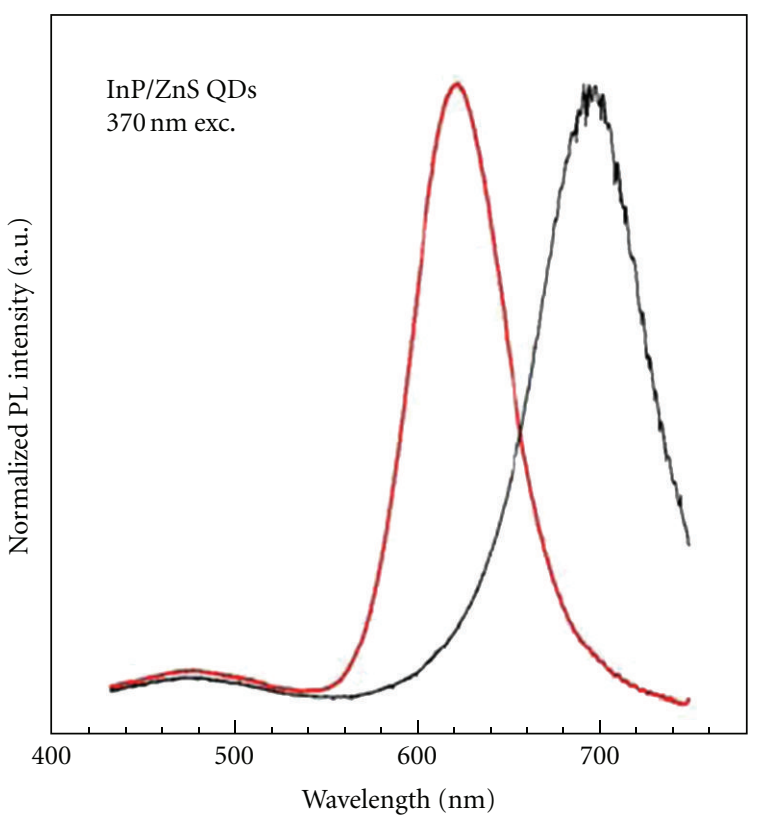

FIGURE 3: Steady-state photoluminescence spectra of two different sized InP/ZnS QD samples under the $370 \mathrm{~nm}$ excitation (reprinted with permission from [42], Copyright 2009, IOP Publishing).

The molar ratios of indium to phosphorus of $2: 1$ give the best quality InP nanocrystals when the noncoordinating octadecene is used as a solvent $[43,44]$. When the ratios of the metal to ligand are above or below this ratio, the reaction gives nanocrystals with no distinguishable UV-Vis spectral features, implying that the size distribution is broad. The use of the $\mathrm{ZnS}$ coating layer gives the red-shift in both the absorption and PL spectra of about 2-3 nm (Figure 4) as well as an improvement in the PL QY from $<1$ to $20 \%$ [44]. 


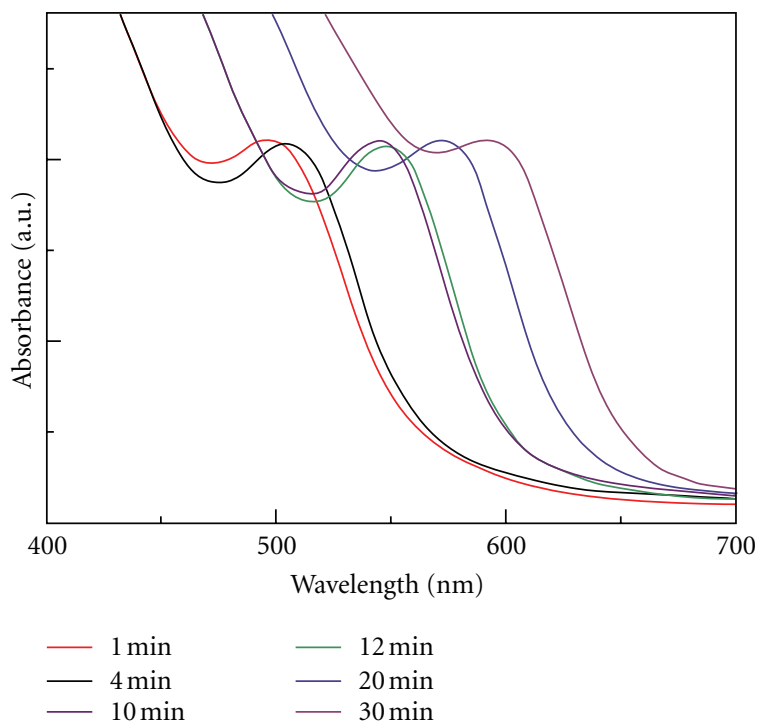

(a)

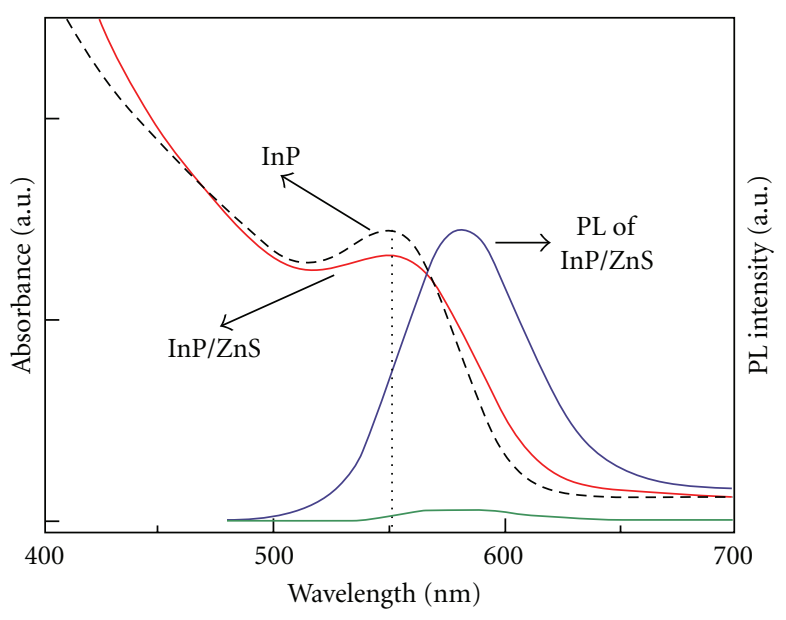

(b)

FIgURE 4: (a) Temporal evolution of the UV-Vis spectra of InP nanocrystals in ODE. (b) UV-Vis spectra of InP nanocrystals before (black dashed) and after (red solid) passivation with ZnS and PL spectra of InP (green solid) and InP/ZnS nanocrystals (blue solid) (reprinted with permission from [44], Copyright 2008, American Chemical Society).

Myristic and palmitic acids, which are fatty acids of intermediate chain length, serve as the best ligands for controlled nucleation growth rates that yield relatively monodisperse nanocrystals when the ratio of the fatty acid to indium is $3: 1$ [43]. Uniquely, a "green" approach where by high-quality InP nanocrystals are synthesized without the addition of a coordinating solvent or ligands, using indium carboxylates as precursors for indium, was reported [45]. The carboxylate ions act as the in situ coordination ligands. The usage of weakly coordinating fatty acid esters in the preparation of high-quality InP QDs is known [46]. In these reactions, trimethylindium is used. It readily dissolves in the ester at room temperature and generates a soluble indium-ester complex with a higher activity than the indium carboxylate. The reported reaction pathway for this reaction is given in Scheme 1.

The FWHM of the produced InP QD PL spectra is about $60 \mathrm{~nm}$. When protic reagents such as amines are used, they hydrolyze (TMS) ${ }_{3} \mathrm{P}$ thereby accelerating the reaction with the indium precursors, but reducing the FWHM to around $48 \mathrm{~nm}$. The role of the fatty acids and the protic reagents is mainly to increase the polarity of the reaction media affording better solubility as well as homogeneity of the precursors culminating in rapid but controlled reactions [47]. Polyethylene glycol (PEG) has been used as a weakly coordinating solvent for the one-pot synthesis of InP/ZnS QDs [47]. PEG offers the major advantages that it is nontoxic, inexpensive, and recyclable. The InP/ZnS QDs prepared in the PEG medium have quantum efficiencies as high as 30\% with emissions ranging from 500 to $655 \mathrm{~nm}$ with FWHM of approximately $46 \mathrm{~nm}$. Extremely small InP QDs have also been synthesized in a reaction where the protic reagents are used and heating carried out at 100 $220^{\circ} \mathrm{C}$ for a day [48]. Protière and Reiss also reported the activation effects of amines when they prepared $\operatorname{In}_{2} \mathrm{O}_{3}$ coated InP QDs having an emission profile of $583 \mathrm{~nm}$ and FWHM of 50-60 nm [49]. The shell growth was done in situ without an addition of any precursor but was induced by oleylamine which was added at the start of the reaction. In 2010, Bawendi's group published a mechanism for the formation of InP QDs (Scheme 2) [50], demonstrating that amines inhibited precursor decomposition, contrary to the aforementioned reports that they are activating agents in the synthesis.

According to the scheme, amine inhibition resulted from solvation effects associated with steps that lead to the formation of complexes 1 and 2. Both the amine and $(\mathrm{TMS})_{3} \mathrm{P}$ compete for the same indium metal centre and hence an increase in the concentration of the amine decreases the formation of complex 1 as the sterically hindered phosphine precursor is less likely to approach the metal centre. During the nucleation step, molecular phosphorus precursors are completely used up. As a result, subsequent growth in nanocrystal sizes is exclusively due to ripening from nonmolecular InP species giving rise to broad size distributions.

$\mathrm{Li}$ and coworkers [51] reported a low-temperature solvothermal synthesis of InP nanocrystals that gave a broad size distribution due to a lack of separation of the nucleation and growth steps. UV irradiation of the nanocrystals in the presents of $\mathrm{Zn}^{2+}$ ions and thioglycolic acid yielded a thick $\mathrm{ZnS}$ shell and improved their PL efficiencies (30$68 \%)$. When the HF etching of the InP cores preceded the $\mathrm{ZnS}$ coating step, a blue shift in the absorption spectra was observed and this was ascribed to a slight QD size reduction during the etching process, while the observed improvement in the photoluminescence was due to a partial surface oxidation of the InP QD surfaces [17]. 


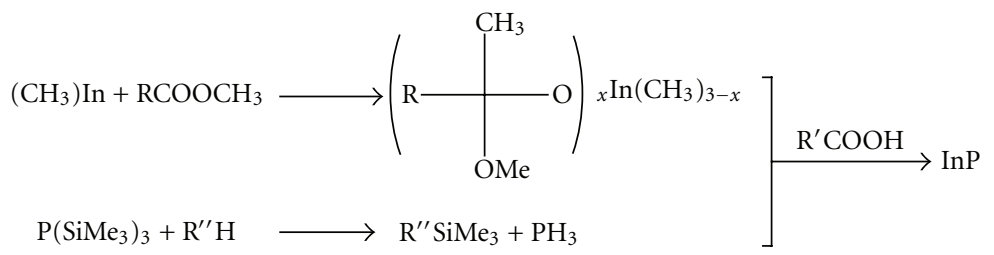

$\mathrm{R}=\mathrm{CH}_{3}\left(\mathrm{CH}_{2}\right)_{x} \quad \mathrm{R}^{\prime}=\mathrm{CH}_{3}\left(\mathrm{CH}_{2}\right)_{x} \mathrm{CH}_{2} \quad \mathrm{R}^{\prime \prime}=\mathrm{CH}_{3}\left(\mathrm{CH}_{2}\right)_{x} \mathrm{CH}_{2} \mathrm{COO}$ or $\left(\mathrm{CH}_{3}\left(\mathrm{CH}_{2}\right)_{x} \mathrm{CH}_{2}\right) \mathrm{N}$

Scheme 1: Proposed reaction scheme through an indium-ester intermediate (reprinted with permission from [46], Copyright 2006, American Chemical Society).

$\left[\operatorname{In}(\mathrm{MA})_{3}\right] \cdot\left(\mathrm{NH}_{2}\right)_{n}+(\mathrm{TMS})_{3} \mathrm{P} \longrightarrow[\mathrm{InP}]+3 \mathrm{TMS}-\mathrm{X}$

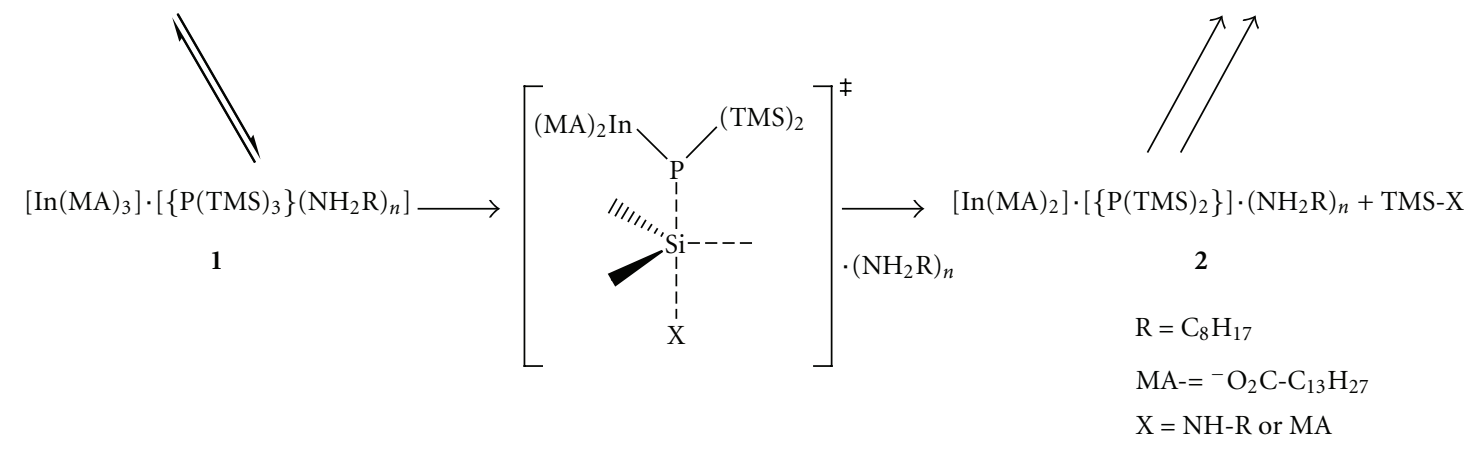

Scheme 2: Proposed mechanism for amine inhibited InP synthesis (reprinted with permission from [50], Copyright 2010, Wiley-VCH Verlag $\mathrm{GmbH}$ and Co. KGaA).

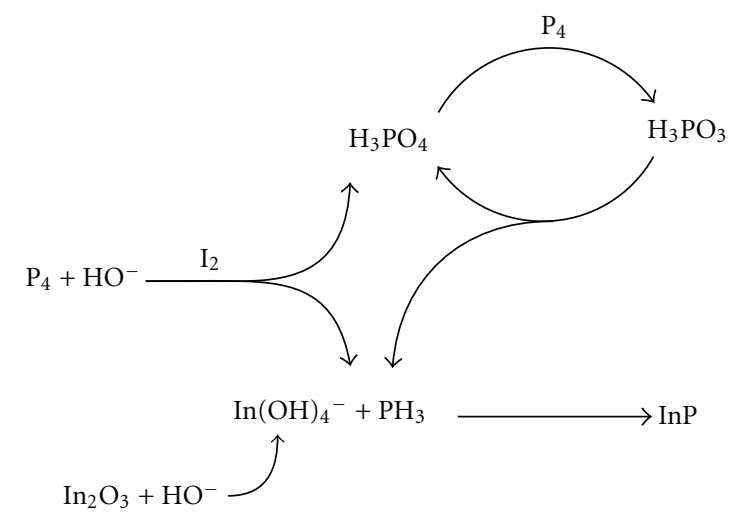

Scheme 3: Growth pathway to InP QDs adapted from the GaP scheme [52].

Another green chemistry approach recently documented involves the usage of an aqueous synthetic route to InP QDs. The system uses an indium oxide, $\operatorname{In}_{2} \mathrm{O}_{3}$, and white phosphorus $\mathrm{P}_{4}$ for the synthesis of the InP core [52]. The formation of the InP nanocrystals (Scheme 3) was confirmed by both the X-ray photoelectron spectroscopy and energydispersive $\mathrm{X}$-ray (EDX) analyses that gave $\mathrm{In}: \mathrm{P}$ ratios of $1: 1.02$ and $1: 1.01$, respectively.

However, this synthetic route produces larger-sized nanocrystals with poor size distribution.

Yet another approach by Green and O'Brien involves the pyrolysis of a single source precursor $\operatorname{In}\left(\mathrm{PBu}_{2}{ }^{t}\right)_{3}$ in 4ethylpyridine at $167^{\circ} \mathrm{C}$ to give large InP QDs [53]. The nanocrystals obtained were about $7 \mathrm{~nm}$ in diameter with the PL spectrum exhibiting a broad near band edge luminescence peak at $534 \mathrm{~nm}$. No QY data was reported here. A coreduction colloidal method for the synthesis of relatively high-quality InP nanocrystals using indium acetate, $\mathrm{PCl}_{3}$, and a superhydride $\operatorname{LiBH}\left(\mathrm{C}_{2} \mathrm{H}_{5}\right)_{3}$ and stearic acid is equally known [54]. In this heating-up type synthesis in the presence of noncoordinating octadecene, the simultaneous reduction of the indium and phosphorus precursors was carried out at low temperature followed by the elevation of the temperature to around $250-270^{\circ} \mathrm{C}$ for growth of the InP nanocrystals. The PL spectra containing both a high-energy band edge and a low-energy surface trap-related emission were obtained. 


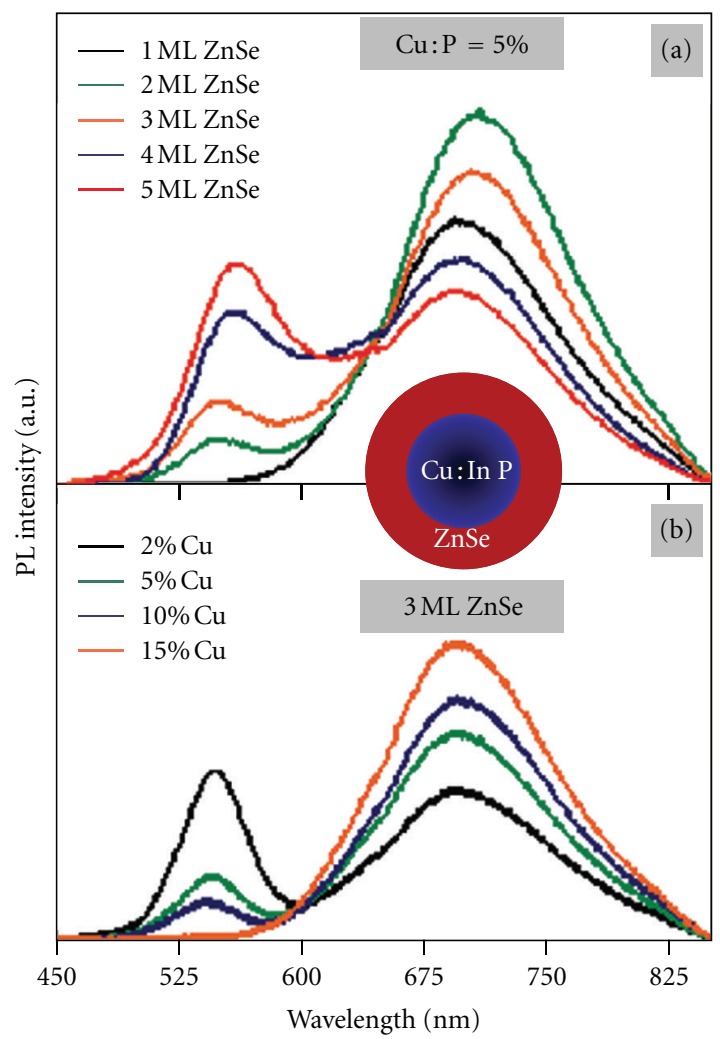

Figure 5: PL spectra of $\mathrm{Cu}: \mathrm{InP} \mathrm{d}$-dots (5\% of $\mathrm{Cu}: \mathrm{P}$ precursor ratio) with different thicknesses of ZnSe shells (a) and PL spectra of $\mathrm{Cu}: \mathrm{InP} / \mathrm{ZnSe} \mathrm{QDs}$ formed with different $\mathrm{Cu}: \mathrm{P}$ precursor ratios (b) (reprinted with permission from [60], Copyright 2009, American Chemical Society).

Posttreatment of the cores with the etchant HF resulted in the fluorescence intensity increasing by a factor of 80 and the PL efficiency improved from about $0.25 \%$ to about $20 \%$.

4.2. Alloying and Doping of the InP QDs. Most of the prepared QDs are binary in nature. The binary quantum dots have the size of the nanocrystals, and hence the emission profile controlled by a systematic adjustment of the reaction parameters, namely, temperature, time, and concentration of both the precursors and surfactant molecules. The emission profiles of alloyed III-V nanocrystals can be fine-tuned using composition as well, allowing them to emit in the near-infrared region. One such system, InAs $\mathrm{P}_{1-x} / \mathrm{InP} / \mathrm{ZnSe}$, with a PL QY of about 3.5\%, has been reported [55]. Another alloyed QD system, InGaP, was synthesized using a microwave-enhanced method [56]. QYs of about 15\% were obtained prior to etching and improved to $68 \%$ upon etching due to the passivation of surface states. Few literature reports on the doping of InP QDs are due to the low solubility of the dopants in the III-V semiconductor matrix [57, 58]. Manganese-doped InP QDs were obtained by using the hightemperature dehalodesilylation and injection methods in a coordinating solvent. The PL studies showed only small differences between the doped and pure samples. In another report, InP : Mn QDs gave a PL spectrum with a maximum peak at $570 \mathrm{~nm}$ and an FWHM of $110 \mathrm{~nm}$. However, it was not feasible to estimate the contribution of the dopant to the overall PL property of this system [59]. Copper-doped InP/ZnSe (Cu:InP/ZnSe) QDs have also been reported, exhibiting emission profiles that could be tuned from 630 to $1100 \mathrm{~nm}$, thus covering the critical near-infrared (NIR) window for biomedical applications [60]. In these copperdoped QDs, the band edge emission from InP is eliminated (Figure 5), and pure dopant emission, with PL QYs of up to $40 \%$, is attained.

\section{InP QDs Applied in Biomedical Research}

The most recent major application of QDs lies in the area of biomedicine. One critical requirement for the use of luminescent QDs in biotechnology and medicine requires that the nanocrystals be compatible with the aqueous media [11]. To address this issue, various strategies have been developed such as introducing bifunctional hydrophilic capping ligands which include ligand exchange reactions. When solubilization of the QDs is attained, the QDs can then be further functionalized by conjugation to a number of biological molecules that include targeting peptides or antibodies. There are three modes of conjugating biomolecules to QDs which are commonly employed (a) covalent attachment, (b) direct attachment of biomolecules to the surface of the QDs, and (c) noncovalent electrostatic interaction. The discussion 

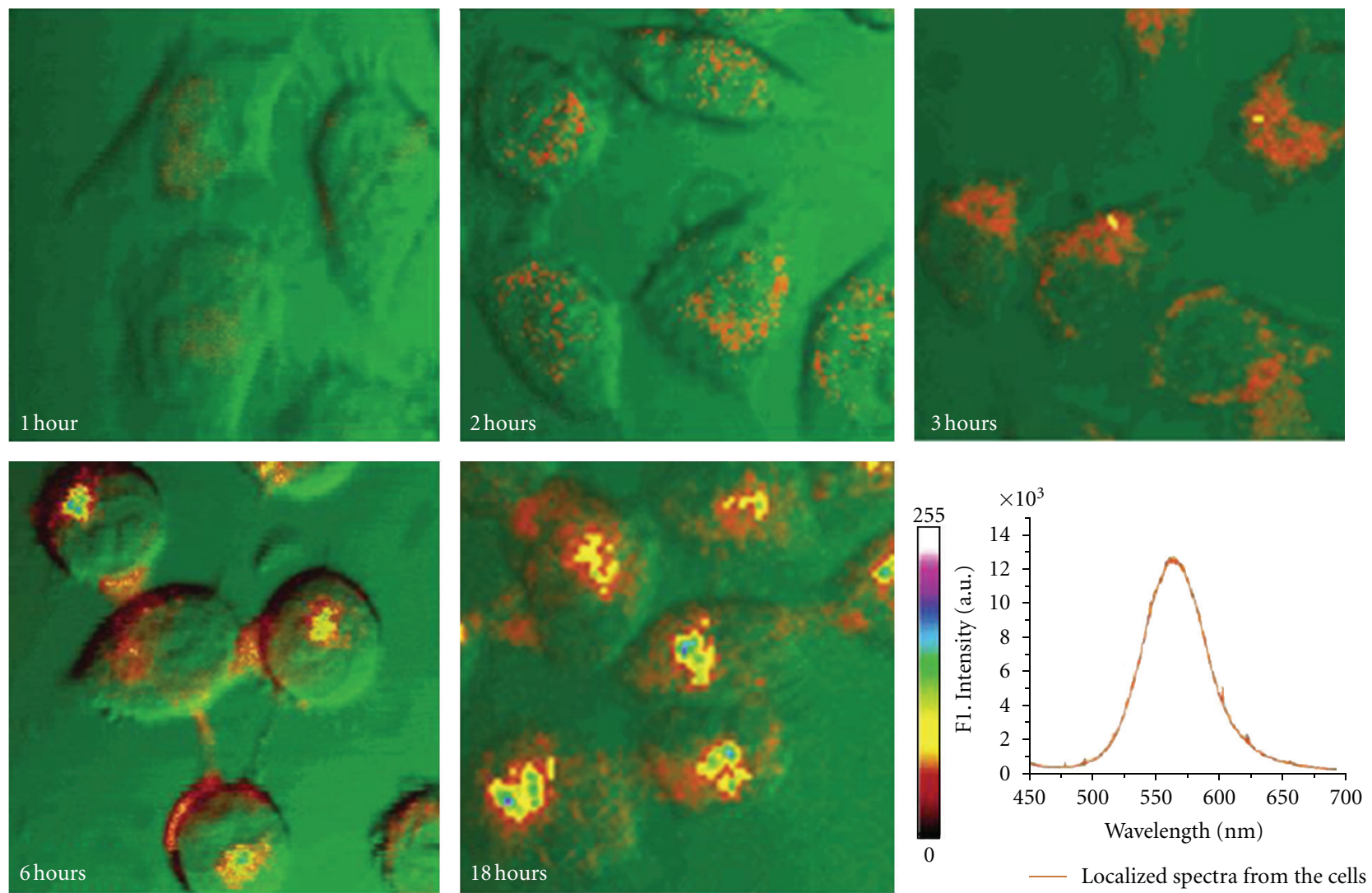

FIGURE 6: Confocal images showing fluorescence (intensity coded red-white channels) of QD-FA in MVBs of KB cells and a localized PL emission spectrum (reprinted with permission from [14], Copyright 2005, American Chemical Society).

of these methods can be found in the review by Sapsford et al. [61].

There are reports in the literature on the application of indium-based quantum dots in biological systems. The use of folic acid in the biofunctionalization of mercaptoacetic acid-capped InP/ZnS for use in the folate-receptormediated delivery was reported [14]. The folate-receptors are overexpressed in human cancerous cells which include the malignancies of the kidney, ovary, or prostate. The uptake of the QD-FA conjugates in the KB cells was confirmed by their rapid uptake which was immediately followed by the formation of multivesicular bodies (MVBs). Competition experiments in which the KB cells were first saturated with excess folic acid and then subsequently incubated with the QD conjugates confirmed the receptor-mediated delivery of the QDs (Figure 6).

Mercaptosuccinic acid-capped InP/ZnS QDs have also been used in the imaging of human pancreatic cancer cells (MiaPaCa or Panc-1) [15]. These QDs were first bioconjugated to pancreatic cancer targeting monoclonal antibodies anticlaudin 4 and antiprostate stem cell antigen (antiPSCA) whose corresponding antigens are well known to be overexpressed in primary and metastatic pancreatic cancer. Cellular uptake of the QD bioconjugates was observed from the optical signal of the MiaPaCa cells as shown in Figure 7.
There were no signs of morphological damage to the cells, showing the low cytotoxicity of the indium-based QDs which could make the usage of indium-based QDs highly favourable for biological applications.

\section{Conclusions}

A lot of progress has been registered in the preparation of high-quality InP QDs. These systems have been best prepared by using coordinating and noncoordinating solvents. Other reports of fully applying "green chemistry" have also been documented. The PL QY properties of these systems can be largely improved by employing the core/shell strategy. The FWHM values ranging from 50 to $100 \mathrm{~nm}$ are still broad in comparison with the long known cadmium-containing QD systems. This clearly demonstrates that the QDs lack one major quality of being monodispersed in comparison to the II-VI systems. Research on improving their optical properties is ongoing. Furthermore, the development of alloyed QDs based on InP which allow for fine-tuning the emission profiles without altering particle size ushers in new possibilities in designing near-infrared emitting probes for in vivo molecular imaging.

The near-infrared emitting probes have the main advantage of being able to overcome problems of autofluorescence 


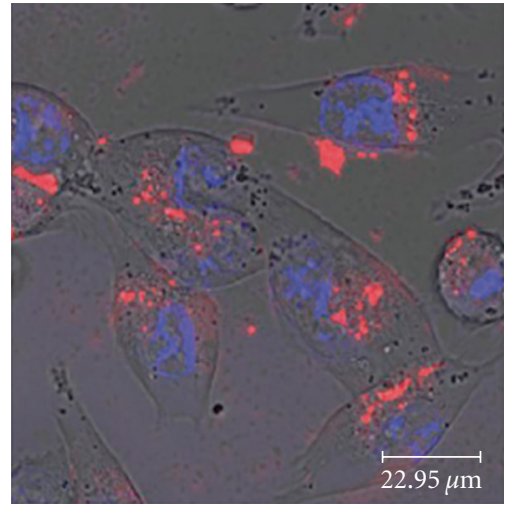

(a)

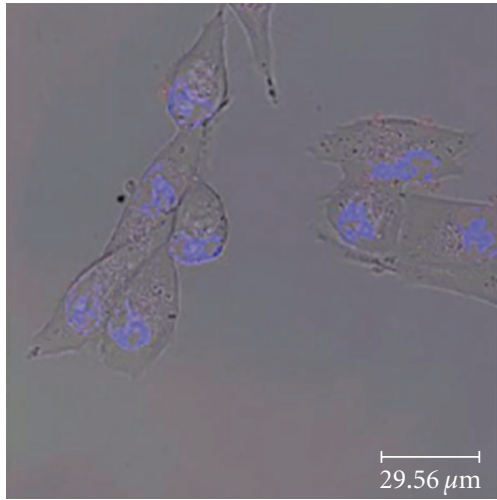

(b)

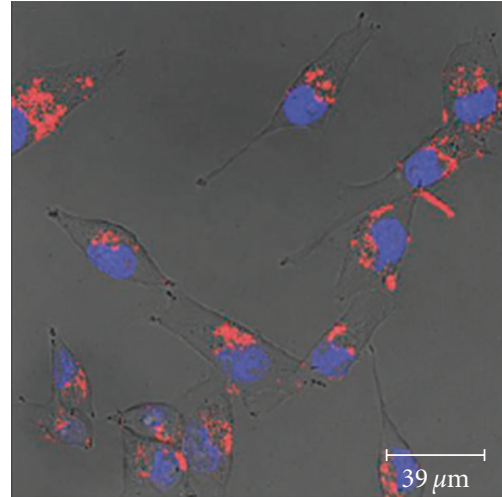

(c)

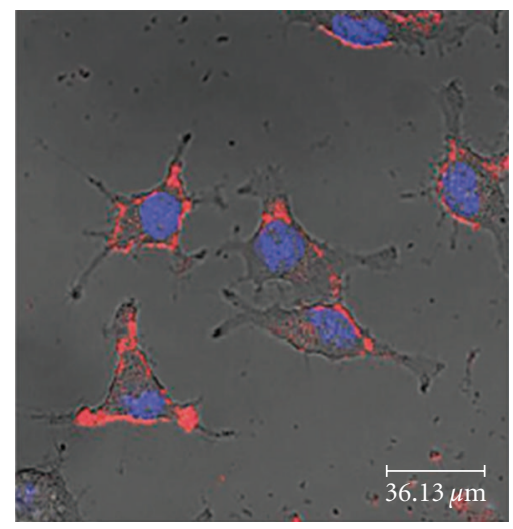

(d)

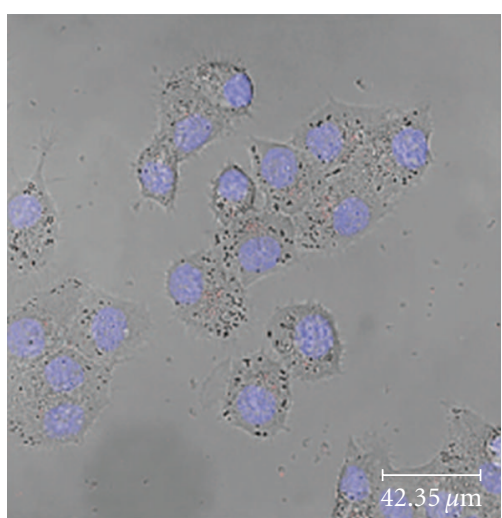

(e)

FIgure 7: Confocal microscopic images of (a) MiaPaCa cells treated with anticlaudin 4-conjugated InP/ZnS QDs, (b) MiaPaCa cells treated with unconjugated InP/ZnS QDs, (c) MiaPaCa cells treated with anti-PSCA-conjugated InP/ZnS QDs, (d) XPA3 cells treated with anticlaudin 4-conjugated InP/ZnS QDs, and (e) KB cells treated with anticlaudin 4-conjugated InP/ZnS QDs. Red and blue represent emissions from InP/ZnS QDs and Hoechst 33342, respectively (reprinted with permission from [15], Copyright 2009, American Chemical Society).

from biological materials. The latest demonstration of the use of biofunctionalized InP/ZnS QDs raised interest in the development of future nanoprobes for biomedical applications. The nanoprobes would be a more welcomed replacement of the highly toxic cadmium-based QD systems that have dominated current bioimaging studies.

\section{References}

[1] K. H. Lee, "Quantum dots: a quantum jump for molecular imaging?” Journal of Nuclear Medicine, vol. 48, no. 9, pp. 14081410, 2007.

[2] V. I. Klimov, "Nanocrystal quantum dots: from fundamental photophysics to multicolor lasing," Los Alamos Science, no. 28, pp. 214-220, 2003.

[3] A. P. Alivisatos, "Semiconductor clusters, nanocrystals, and quantum dots," Science, vol. 271, no. 5251, pp. 933-937, 1996.

[4] F. Pinaud, X. Michalet, L. A. Bentolila et al., "Advances in fluorescence imaging with quantum dot bio-probes," Biomaterials, vol. 27, no. 9, pp. 1679-1687, 2006.

[5] P. Reiss, M. Protière, and L. Li, "Core/shell semiconductor nanocrystals," Small, vol. 5, no. 2, pp. 154-168, 2009.

[6] K. E. Andersen, C. Y. Fong, and W. E. Pickett, "Quantum confinement in CdSe nanocrystallites," Journal of Non-Crystalline Solids, vol. 299-302, no. 2, pp. 1105-1110, 2002.
[7] U. Resch-Genger, M. Grabolle, S. Cavaliere-Jaricot, R. Nitschke, and T. Nann, "Quantum dots versus organic dyes as fluorescent labels," Nature Methods, vol. 5, no. 9, pp. 763-775, 2008.

[8] M. V. Artemyev, U. Woggon, R. Wannemacher, H. Jaschinski, and W. Langbein, "Light trapped in a photonic dot: microspheres act as a cavity for quantum dot emission," Nano Letters, vol. 1, no. 6, pp. 309-314, 2001.

[9] N. P. Gaponik, D. V. Talapin, A. L. Rogach, and A. Eychmuller, "Electrochemical synthesis of CdTe nanocrystal/polypyrrole composites for optoelectronic applications," Journal of Materials Chemistry, vol. 10, no. 9, pp. 2163-2166, 2000.

[10] M. Bruchez Jr., M. Moronne, P. Gin, S. Weiss, and A. P. Alivisatos, "Semiconductor nanocrystals as fluorescent biological labels," Science, vol. 281, no. 5385, pp. 2013-2016, 1998.

[11] W. C. W. Chan and S. Nie, "Quantum dot bioconjugates for ultrasensitive nonisotopic detection," Science, vol. 281, no. 5385, pp. 2016-2018, 1998.

[12] X. Michalet, F. F. Pinaud, L. A. Bentolila et al., "Quantum dots for live cells, in vivo imaging, and diagnostics," Science, vol. 307, no. 5709, pp. 538-544, 2005.

[13] A. M. Derfus, W. C. W. Chan, and S. N. Bhatia, "Probing the cytotoxicity of semiconductor quantum dots," Nano Letters, vol. 4, no. 1, pp. 11-18, 2004. 
[14] D. J. Bharali, D. W. Lucey, H. Jayakumar, H. E. Pudavar, and P. N. Prasad, "Folate-receptor-mediated delivery of InP quantum dots for bioimaging using confocal and two-photon microscopy," Journal of the American Chemical Society, vol. 127, no. 32, pp. 11364-11371, 2005.

[15] K. T. Yong, H. Ding, I. Roy et al., "Imaging pancreatic cancer using bioconjugated inp quantum dots," ACS Nano, vol. 3, no. 3, pp. 502-510, 2009.

[16] L. Langof, E. Ehrenfreund, E. Lifshitz, O. I. Micic, and A. J. Nozik, "Continuous-wave and time-resolved optically detected magnetic resonance studies of nonetched/etched InP nanocrystals," Journal of Physical Chemistry B, vol. 106, no. 7, pp. 1606-1612, 2002.

[17] H. J. Byun, J. C. Lee, and H. Yang, "Solvothermal synthesis of InP quantum dots and their enhanced luminescent efficiency by post-synthetic treatments," Journal of Colloid and Interface Science, vol. 355, no. 1, pp. 35-41, 2011.

[18] T. Puangmali, M. Califano, and P. Harrison, "Monotonic evolution of the optical properties in the transition from three- to quasi-two-dimensional quantum confinement in InAs nanorods," Journal of Physical Chemistry $C$, vol. 114, no. 15, pp. 6901-6908, 2010.

[19] K. Zhu, J. Shi, and L. Zhang, "Preparation and optical absorption of InSb microcrystallites embedded in $\mathrm{SiO}_{2}$ thin films," Solid State Communications, vol. 107, no. 2, pp. 79-84, 1998.

[20] R. S. Yadav, P. Mishra, R. Mishra, M. Kumar, and A. C. Pandey, "Growth mechanism and optical property of CdS nanoparticles synthesized using amino-acid histidine as chelating agent under sonochemical process," Ultrasonics Sonochemistry, vol. 17, no. 1, pp. 116-122, 2010.

[21] K. Kyhm, J. H. Kim, S. M. Kim, and H. S. Yang, "Gain dynamics and excitonic transition in CdSe colloidal quantum dots," Optical Materials, vol. 30, no. 1, pp. 158-160, 2007.

[22] T. Rajh, O. I. Mićić, and A. J. Nozik, "Synthesis and characterization of surface-modified colloidal CdTe quantum dots," Journal of Physical Chemistry, vol. 97, no. 46, pp. 11999-12003, 1993.

[23] S. Ramanathan, S. Patibandla, S. Bandyopadhyay, J. Anderson, and J. D. Edwards, "Fluorescence spectroscopy of electrochemically self-assembled ZnSe and Mn:ZnSe nanowires," Nanotechnology, vol. 19, no. 19, Article ID 195601, 2008.

[24] Y. Yin and A. P. Alivisatos, "Colloidal nanocrystal synthesis and the organic-inorganic interface," Nature, vol. 437, no. 7059, pp. 664-670, 2005.

[25] Y. W. Cao and U. Banin, "Growth and properties of semiconductor core/shell nanocrystals with InAs cores," Journal of the American Chemical Society, vol. 122, no. 40, pp. 9692-9702, 2000.

[26] P. Mohapatra, M. X. Dung, J.-K. Choi, S. Jeong, and H.D. Jeong, "Effects of curing temperature on the optical and charge trap properties of InP quantum dot thin films," Bulletin of the Korean Chemical Society, vol. 32, no. 1, pp. 263-272, 2011.

[27] J. Lim, W. K. Bae, D. Lee et al., “Inp@znses, core@composition gradient shell quantum dots with enhanced stability," Chemistry of Materials, vol. 23, no. 20, pp. 4459-4463, 2011.

[28] M. R. Kim, J. H. Chung, M. Lee, S. Lee, and D. J. Jang, "Fabrication, spectroscopy, and dynamics of highly luminescent core-shell InP@ZnSe quantum dots," Journal of Colloid and Interface Science, vol. 350, no. 1, pp. 5-9, 2010.

[29] J. L. Blackburn, R. J. Ellingson, O. I. Mićić, and A. J. Nozik, "Electron relaxation in colloidal InP quantum dots with photogenerated excitons or chemically injected electrons,"
Journal of Physical Chemistry B, vol. 107, no. 1, pp. 102-109, 2003.

[30] O. I. Mićić, J. Sprague, Z. Lu, and A. J. Nozik, "Highly efficient band-edge emission from InP quantum dots," Applied Physics Letters, vol. 68, no. 22, pp. 3150-3152, 1996.

[31] D. V. Talapin, N. Gaponik, H. Borchert, A. L. Rogach, M. Haase, and H. Weller, "Etching of colloidal InP nanocrystals with fluorides: photochemical nature of the process resulting in high photoluminescence efficiency," Journal of Physical Chemistry B, vol. 106, no. 49, pp. 12659-12663, 2002.

[32] P. Reiss, "ZnSe based colloidal nanocrystals: synthesis, shape control, core/shell, alloy and doped systems," New Journal of Chemistry, vol. 31, no. 11, pp. 1843-1852, 2007.

[33] C. B. Murray, D. J. Norris, and M. G. Bawendi, "Synthesis and characterization of nearly monodisperse CdE $(\mathrm{E}=\mathrm{S}$, Se, Te) semiconductor nanocrystallites," Journal of the American Chemical Society, vol. 115, no. 19, pp. 8706-8715, 1993.

[34] G. K. Soon, Y. Piao, J. Park et al., "Kinetics of monodisperse iron oxide nanocrystal formation by "heating-up" process," Journal of the American Chemical Society, vol. 129, no. 41, pp. 12571-12584, 2007.

[35] L. Li and P. Reiss, "One-pot synthesis of highly luminescent InP/ZnS nanocrystals without precursor injection," Journal of the American Chemical Society, vol. 130, no. 35, pp. 1158811589, 2008.

[36] O. I. Mićić, C. J. Curtis, K. M. Jones, J. R. Sprague, and A. J. Nozik, "Synthesis and characterization of InP quantum dots," Journal of Physical Chemistry, vol. 98, no. 19, pp. 4966-4969, 1994.

[37] O. I. Mićić and A. J. Nozik, "Synthesis and characterization of binary and ternary III-V quantum dots," Journal of Luminescence, vol. 70, no. 1-6, pp. 95-107, 1996.

[38] O. I. Micic, B. B. Smith, and A. J. Nozik, "Core-shell quantum dots of lattice-matched $\mathrm{ZnCdSe}_{2}$ shells on $\mathrm{InP}$ cores: experiment and theory," Journal of Physical Chemistry B, vol. 104, no. 51, pp. 12149-12156, 2000.

[39] S. Kim, J. Park, S. Kim, W. Jung, J. Sung, and S. W. Kim, “The effects of staggered bandgap in the InP/CdSe and CdSe/InP core/shell quantum dots," Journal of Colloid and Interface Science, vol. 346, no. 2, pp. 347-351, 2010.

[40] E. Ryu, S. Kim, E. Jang et al., "Step-wise synthesis of InP/ZnS Core-Shell quantum dots and the role of zinc acetate," Chemistry of Materials, vol. 21, no. 4, pp. 573-575, 2009.

[41] L. Li, M. Protière, and P. Reiss, "Economic synthesis of high quality InP nanocrystals using calcium phosphide as the phosphorus precursor," Chemistry of Materials, vol. 20, no. 8, pp. 2621-2623, 2008.

[42] P. T. Thuy, U. T. D. Thuy, T. T. K. Chi et al., "Time-resolved photoluminescence measurements of InP/ZnS quantum dots," Journal of Physics, vol. 187, Article ID 012014, 2009.

[43] D. Battaglia and X. Peng, "Formation of high quality InP and InAs nanocrystals in a noncoordinating solvent," Nano Letters, vol. 2, no. 9, pp. 1027-1030, 2002.

[44] A. Narayanaswamy, L. F. Feiner, and P. J. Van Der Zaag, "Temperature dependence of the photoluminescence of InP/ZnS quantum dots," Journal of Physical Chemistry C, vol. 112, no. 17, pp. 6775-6780, 2008.

[45] D. W. Lucey, D. J. MacRae, M. Furis, Y. Sahoo, A. N. Cartwright, and P. N. Prasad, "Monodispersed InP quantum dots prepared by colloidal chemistry in a noncoordinating solvent," Chemistry of Materials, vol. 17, no. 14, pp. 3754-3762, 2005. 
[46] S. Xu, S. Kumar, and T. Nann, "Rapid synthesis of high-quality InP nanocrystals," Journal of the American Chemical Society, vol. 128, no. 4, pp. 1054-1055, 2006.

[47] S. Hussain, N. Won, J. Nam, J. Bang, H. Chung, and S. Kim, "One-pot fabrication of high-quality InP/ZnS (Core/Shell) quantum dots and their application to cellular imaging," ChemPhysChem, vol. 10, no. 9-10, pp. 1466-1470, 2009.

[48] O. I. Micic, S. P. Ahrenkiel, and A. J. Nozik, "Synthesis of extremely small InP quantum dots and electronic coupling in their disordered solid films," Applied Physics Letters, vol. 78, no. 25, pp. 4022-4024, 2001.

[49] M. Protière and P. Reiss, "Amine-induced growth of an $\mathrm{In}_{2} \mathrm{O}_{3}$ shell on colloidal InP nanocrystals," Chemical Communications, no. 23, pp. 2417-2419, 2007.

[50] P. M. Allen, B. J. Walker, and M. G. Bawendi, "Mechanistic insights into the formation of InP quantum dots," Angewandte Chemie, vol. 49, no. 4, pp. 760-762, 2010.

[51] C. Li, M. Ando, H. Enomoto, and N. Murase, "Highly luminescent water-soluble $\mathrm{InP} / \mathrm{ZnS}$ nanocrystals prepared via reactive phase transfer and photochemical processing," Journal of Physical Chemistry C, vol. 112, no. 51, pp. 20190-20199, 2008.

[52] S. Gao, J. Lu, N. Chen, Y. Zhao, and Y. Xie, "Aqueous synthesis of III-V semiconductor GaP and InP exhibiting pronounced quantum confinement," Chemical Communications, vol. 8, no. 24, pp. 3064-3065, 2002.

[53] M. Green and P. O'Brien, “A novel metalorganic route for the direct and rapid synthesis of monodispersed quantum dots of indium phosphide," Chemical Communications, no. 22, pp. 2459-2460, 1998.

[54] Z. Liu, A. Kumbhar, D. Xu, J. Zhang, Z. Sun, and J. Fang, "Coreduction colloidal synthesis of III-V nanocrystals: the case of InP," Angewandte Chemie, vol. 47, no. 19, pp. 35403542, 2008.

[55] S. W. Kim, J. P. Zimmer, S. Ohnishi, J. B. Tracy, J. V. Frangioni, and M. G. Bawendi, "Engineering InAsxP1-x/InP/ZnSe III-V alloyed core/shell quantum dots for the near-infrared," Journal of the American Chemical Society, vol. 127, no. 30, pp. 1052610532, 2005.

[56] J. A. Gerbec, D. Magana, A. Washington, and G. F. Strouse, "Microwave-enhanced reaction rates for nanoparticle synthesis," Journal of the American Chemical Society, vol. 127, no. 45, pp. 15791-15800, 2005.

[57] K. Somaskandan, G. M. Tsoi, L. E. Wenger, and S. L. Brock, "Isovalent doping strategy for manganese introduction into III-V diluted magnetic semiconductor nanoparticles: InP:Mn," Chemistry of Materials, vol. 17, no. 5, pp. 1190-1198, 2005.

[58] D. Mocatta, G. Cohen, J. Schattner, O. Millo, E. Rabani, and U. Banin, "Heavily doped semiconductor nanocrystal quantum dots," Science, vol. 332, no. 6025, pp. 77-81, 2011.

[59] Y. Sahoo, P. Poddar, H. Srikanth, D. W. Lucey, and P. N. Prasad, "Chemically fabricated magnetic quantum dots of InP:Mn," Journal of Physical Chemistry B, vol. 109, no. 32, pp. 1522115225, 2005.

[60] R. Xie and X. Peng, "Synthesis of Cu-doped InP nanocrystals (d-dots) with ZnSe diffusion barrier as efficient and colortunable NIR emitters," Journal of the American Chemical Society, vol. 131, no. 30, pp. 10645-10651, 2009.

[61] K. E. Sapsford, T. Pons, I. L. Medintz, and H. Mattoussi, "Biosensing with luminescent semiconductor quantum dots," Sensors, vol. 6, no. 8, pp. 925-953, 2006. 

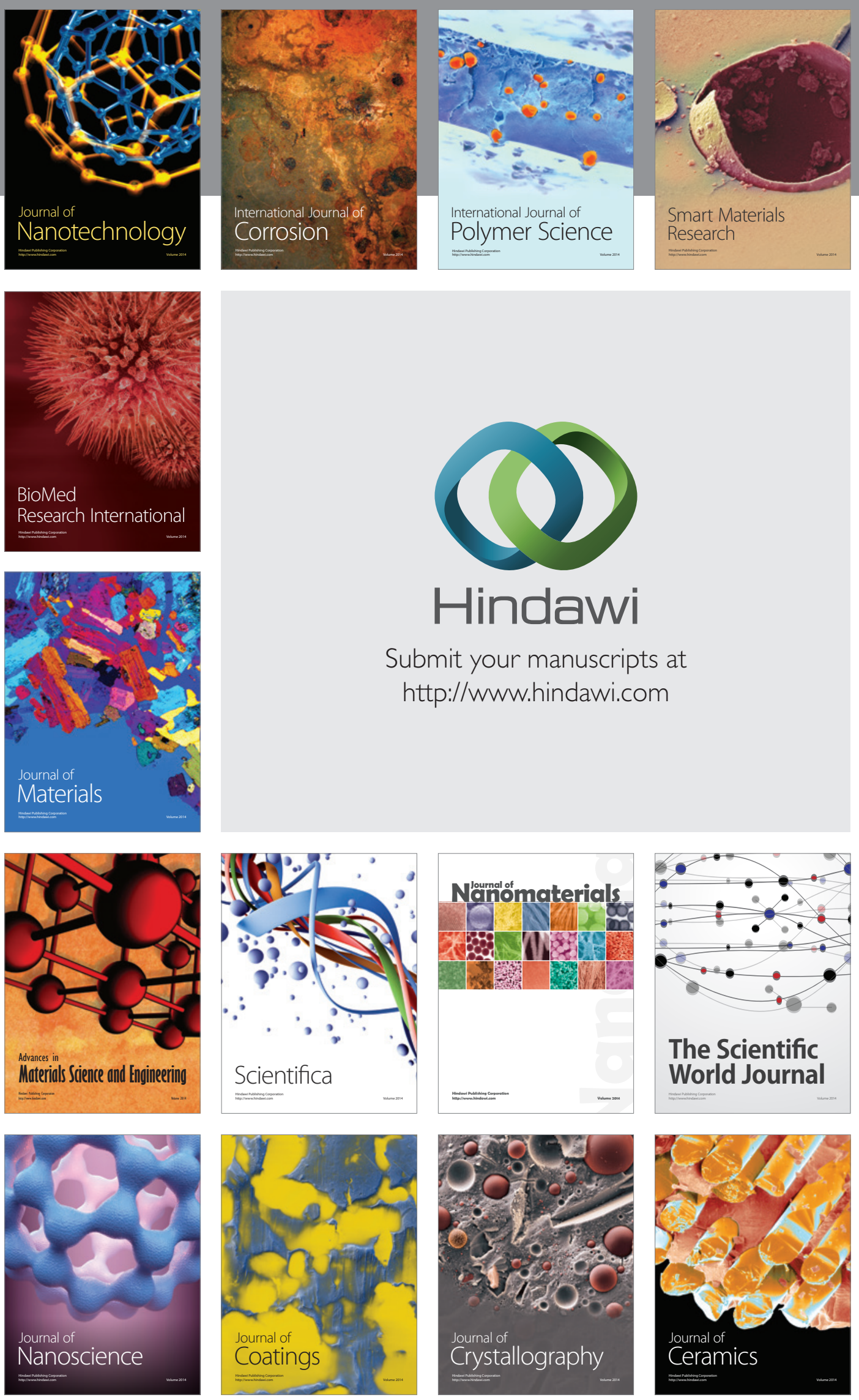

The Scientific World Journal

Submit your manuscripts at

http://www.hindawi.com

\section{World Journal}

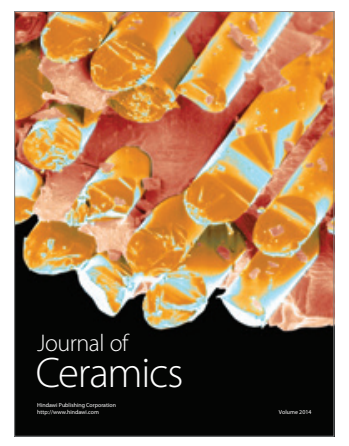

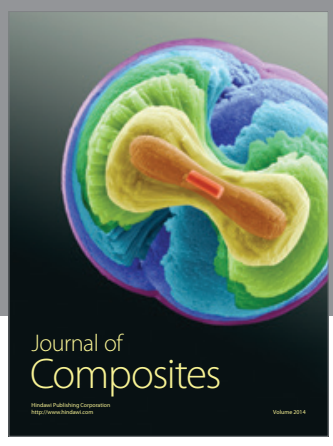
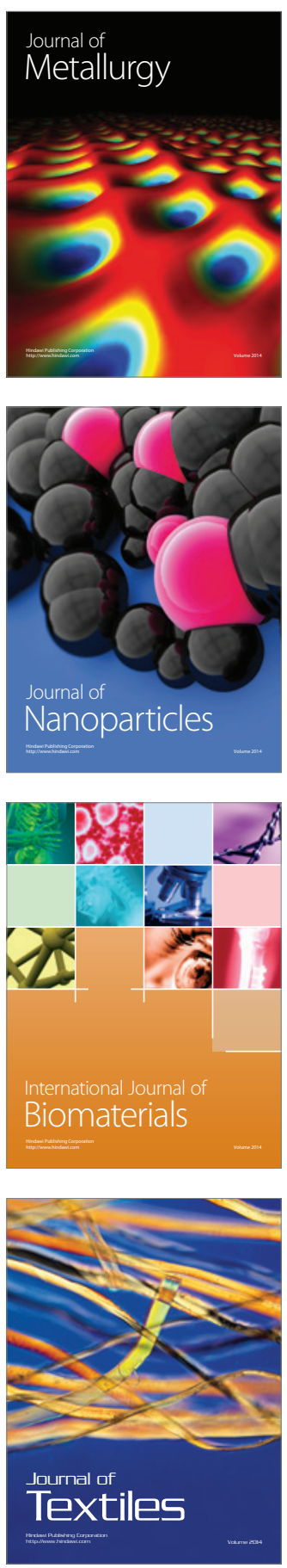